\title{
Degrees that are low for isomorphism
}

\author{
Johanna N.Y. Franklin \\ University of Connecticut \\ Reed Solomon \\ University of Connecticut
}

May 14, 2014

\begin{abstract}
We say that a degree is low for isomorphism if, whenever it can compute an isomorphism between a pair of computable structures, there is already a computable isomorphism between them. We show that while there is no clear-cut relationship between this property and other properties related to computational weakness, the low-forisomorphism degrees contain all Cohen 2-generics and are disjoint from the Martin-Löf randoms. We also consider lowness for isomorphism with respect to the class of linear orders.
\end{abstract}

\section{Introduction}

Within classical computability theory, there are many ways to specify that a particular set $A$ or Turing degree $\mathbf{d}$ is computationally weak. ${ }^{1}$ For example, minimal degrees, low degrees and hyperimmune-free degrees are each computationally weak in an appropriate sense. More recently, there has been considerable interest in sets (or degrees) which are low for $\mathcal{P}$ for various relativizable notions $\mathcal{P}$. Roughly, a set $A$ is low for $\mathcal{P}$ if the relativized notion $\mathcal{P}^{A}$ is the same as $\mathcal{P}$. For example, $A$ is low for Martin-Löf randomness if the collection of sets which are Martin-Löf random relative to $A$ is the same collection of sets which are Martin-Löf random. (See [7] and Chapter 5 of Nies [14] for additional examples and motivation.)

In this paper, we examine a lowness notion in computable model theory which is related to the study of degrees of categoricity. We begin with a summary of definitions from computable model theory to fix our notation.

For a degree $\mathbf{d}$ and computable structures $\mathcal{A}$ and $\mathcal{B}$, we say $\mathcal{A}$ is $\mathbf{d}$-computably isomorphic to $\mathcal{B}$, denoted $\mathcal{A} \cong_{\mathrm{d}} \mathcal{B}$, if there is an isomorphism between $\mathcal{A}$ and $\mathcal{B}$ which is computable from d. If $\mathbf{d}=\mathbf{0}$, we say $\mathcal{A}$ is computably isomorphic to $\mathcal{B}$ and write $\mathcal{A} \cong_{\Delta_{1}^{0}} \mathcal{B}$. A computable structure $\mathcal{A}$ is $\mathbf{d}$-computably categorical if for every computable structure $\mathcal{B}$ which is classically isomorphic to $\mathcal{A}$, we have $\mathcal{A} \cong{ }_{\mathrm{d}} \mathcal{B}$.

\footnotetext{
${ }^{1}$ The authors thank the anonymous referee for helpful comments on this paper.
} 
Definition 1.1. A degree $\mathbf{d}$ is a degree of categoricity if there is a computable structure $\mathcal{A}$ such that $\mathcal{A}$ is $\mathbf{c}$-computably categorical if and only if $\mathbf{c} \geq \mathbf{d}$.

Fokina, Kalimullin and Miller [6] introduced degrees of categoricity. They showed that every degree which is d.c.e. in and above $\mathbf{0}^{(\mathbf{n})}$ is a degree of categoricity and that $\mathbf{0}^{(\omega)}$ is a degree of categoricity. Csima, Franklin and Shore [4] extended these results to show that for every computable ordinal $\alpha, \mathbf{0}^{(\alpha)}$ is a degree of categoricity and for every computable successor ordinal $\alpha$, each degree d.c.e. in and above $\mathbf{0}^{(\alpha)}$ is a degree of categoricity. In the negative direction, Csima, Franklin and Shore proved that every degree of categoricity is hyperarithmetic and hence that there are only countably many degrees of categoricity.

Anderson and Csima [1] continued working in a negative direction and developed several methods to show that certain types of degrees are not degrees of categoricity. In particular, they gave an alternate proof that there are only countably many degrees of categoricity and proved that every noncomputable hyperimmune-free degree is not a degree of categoricity.

More importantly for our current work, Anderson and Csima gave an oracle construction of a noncomputable degree $\mathbf{d} \leq \mathbf{0}^{\prime \prime}$ which is not a degree of categoricity by showing that for every pair of isomorphic computable structures $\mathcal{A}$ and $\mathcal{B}$, if $\mathcal{A} \cong_{\mathrm{d}} \mathcal{B}$, then $\mathcal{A} \cong_{\Delta_{1}^{0}} \mathcal{B}$. Such a degree $\mathbf{d}$ is computationally weak in the sense that $\mathbf{d}$ can only tell that two computable structures are isomorphic when these structures are in fact computably isomorphic. We isolate this property and refer to such degrees as being low for isomorphism.

Definition 1.2. A degree $\mathbf{d}$ is low for isomorphism if for every pair of computable structures $\mathcal{A}$ and $\mathcal{B}, \mathcal{A} \cong_{\mathrm{d}} \mathcal{B}$ if and only if $\mathcal{A} \cong{ }_{\Delta_{1}^{0}} \mathcal{B}$.

Anderson and Csima's oracle construction of a low-for-isomorphism degree can be recast as a forcing construction. In Section 2, we use three different forcing notions to construct lowfor-isomorphism degrees and compare these degrees with other types of computationally weak degrees. In the cases of Mathias forcing and Cohen forcing, the fact that sufficiently generic degrees are low for isomorphism follows from work by Hirschfeldt and Shore [9] and Hirschfeldt, Shore and Slaman [10]. The fact that Sacks forcing also produces low-for-isomorphism degrees does not appear to be in the literature, but it is a minor modification and has been observed by several people. We include a proof here for the sake of completeness.

In Section 3, we give examples of degrees which are not low for isomorphism. In particular, we show that if $\mathbf{d}$ can compute a noncomputable $\Delta_{2}^{0}$ degree or can compute a separating set for a pair of computably inseparable c.e. sets, then $\mathbf{d}$ is not low for isomorphism.

If $\mathbf{d} \neq \mathbf{0}$ is low for isomorphism, then $\mathbf{d}$ is not a degree of categoricity because any computable structure which is $\mathbf{d}$-computable categorical is also computably categorical. However, the converse is not true because the degrees of categoricity are not closed upwards while the degrees which are not low for isomorphism are closed upwards. More specifically, Anderson and Csima show that every noncomputable hyperimmune-free degree is not a degree of categoricity, but it follows from the examples in Section 3 that there are hyperimmune-free degrees which are not low for isomorphism.

In Section 4, we consider the measure of the class of all sets which have low-for-isomorphism degree. Because this class is a Borel tailset, Kolmogorov's 0-1 Law implies that it must have measure 0 or 1. (See Barmpalias, Day and Lewis [2] for background on measure theoretic 
arguments in classical recursion theory.) We show that this class has measure 0 and that no Martin-Löf random degree can be low for isomorphism.

Finally, we will conclude with a brief discussion and some questions.

When working with the notion of lowness for isomorphism, it is convenient to work with computable structures in a fixed computable language rather than considering all computable structures across any computable language.

Proposition 1.3. A degree $\mathbf{d}$ is low for isomorphism if and only if for every pair of computable directed graphs $G_{0}$ and $G_{1}, G_{0} \cong_{\mathbf{d}} G_{1}$ if and only if $G_{0} \cong_{\Delta_{1}^{0}} G_{1}$.

Proof. Hirschfeldt, Khoussainov, Shore and Slinko [8] gave an effective method of coding an arbitrary countable structure $\mathcal{A}$ in a computable language into a countable directed graph $G(\mathcal{A})$ with the following properties.

- $\mathcal{A} \cong \mathcal{B}$ if and only if $G(\mathcal{A}) \cong G(\mathcal{B})$.

- $\mathcal{A}$ is computable if and only if $G(\mathcal{A})$ is computable.

- If $\mathcal{A}$ and $\mathcal{B}$ are computable, then for any Turing degree $\mathbf{d}, \mathcal{A} \cong_{\mathbf{d}} \mathcal{B}$ if and only if $G(\mathcal{A}) \cong_{\mathrm{d}} G(\mathcal{B})$.

The proposition follows immediately from this coding.

Hirschfeldt, Khoussainov, Shore and Slinko actually showed that there are several classes of universal structures in this sense and one could work with any of them in the context of low-for-isomorphism degrees. We choose directed graphs for convenience. However, this restriction to directed graphs raises the natural question of what happens if one restricts to a class of structures (such as linear orders, Boolean algebras or abelian groups) which are not universal in the sense described by Hirschfeldt, Khoussainov, Shore and Slinko [8].

Definition 1.4. Let $\mathcal{C}$ be a class of computable algebraic structures closed under isomorphism within the class of all computable structures. A degree $\mathbf{d}$ is low for $\mathcal{C}$-isomorphism if for every pair of structures $\mathcal{A}, \mathcal{B} \in \mathcal{C}, \mathcal{A} \cong{ }_{\mathrm{d}} \mathcal{B}$ if and only if $\mathcal{A} \cong_{\Delta_{1}^{0}} \mathcal{B}$.

In Section 5, we consider the low-for- $\mathcal{L}$-isomorphism degrees where $\mathcal{L}$ is the class of computable linear orders. We replicate the negative results from Section 3 within this class of degrees, but leave open the question of whether every low-for- $\mathcal{L}$-isomorphism degree is low for isomorphism.

\section{Degrees which are low for isomorphism}

In this section, we use Cohen, Mathias and Sacks forcing to construct low-for-isomorphism degrees. In the cases of Cohen and Mathias forcing, these facts follow immediately from known results about forcing for models of second order arithmetic. We assume familiarity with Cohen and Mathias forcing notions in recursion theory and refer the reader to Jockusch [11] and Cholak, Dzhafarov, Hirst and Slaman [3] for the relevant definitions. Given our 
applications, we sketch the background on models of second order arithmetic in the context of $\omega$-models, although the results hold more generally. Simpson [17] contains a discussion of models of second order arithmetic and forcing in the more general context of these models.

$\mathcal{I} \subseteq \mathcal{P}(\omega)$ is a Turing ideal if $\mathcal{I}$ is closed under Turing reducibility and the Turing join. Given any set $A, \mathcal{I}_{A}=\left\{X \subseteq \omega \mid X \leq_{T} A\right\}$ is a Turing ideal and we refer to $\mathcal{I}_{A}$ as the ideal generated by $A$.

An $\omega$-model $\mathcal{M}$ of $\mathrm{RCA}_{0}$ consists of the standard model of PA (providing the range of the first order variables) together with a Turing ideal $\mathcal{I}_{\mathcal{M}}$ (providing the range of the second order variables). We will abuse notation by equating an $\omega$-model $\mathcal{M}$ of $\mathrm{RCA}_{0}$ with the corresponding ideal $\mathcal{I}_{\mathcal{M}}$. In particular, we say that $\mathcal{M}$ is countable if this ideal is countable, and we let $\mathcal{M}_{A}$ denote the countable $\omega$-model given by the ideal $\mathcal{I}_{A}$.

If $\mathcal{M}$ is an $\omega$-model of $\mathrm{RCA}_{0}$ and $G$ is a set, then $\mathcal{M}[G]$ denotes the smallest $\omega$-model containing $\mathcal{M}$ and the set $G$. That is, the ideal corresponding to $\mathcal{M}[G]$ is the downward closure under $\leq_{T}$ of the set $\{X \oplus G \mid X \in \mathcal{M}\}$. In particular, $\mathcal{M}_{A}[G]=\mathcal{M}_{A \oplus G}$.

Let $\mathcal{M}$ be an $\omega$-model of $\mathrm{RCA}_{0}$. There is a $\Pi_{2}^{0}$ formula $\Phi_{\text {iso }}(X, Y)$ with two free set variables such that for any directed graphs $\mathcal{A}, \mathcal{B} \in \mathcal{M}$ and any function $f \in \mathcal{M}, \mathcal{M} \models \Phi_{\text {iso }}(\mathcal{A} \oplus \mathcal{B}, f)$ if and only if $f$ is an isomorphism from $\mathcal{A}$ to $\mathcal{B}$.

We can now state the relevant facts concerning Cohen and Mathias forcing and apply these facts in our context.

Theorem 2.1. Let $\mathcal{M}$ be a countable $\omega$-model of $\operatorname{RCA}_{0}$ and let $\Phi(X, Y)$ be a $\Sigma_{3}^{0}$ formula with two free set variables such that for some fixed $A \in \mathcal{M}$ there is no $B \in \mathcal{M}$ such that $\mathcal{M} \models \Phi(A, B)$.

(I) (Theorem 3.13, Hirschfeldt, Shore and Slaman [10]) If $G$ is Cohen 2-generic over $\mathcal{M}$, then there is no $B \in \mathcal{M}[G]$ such that $\mathcal{M}[G] \models \Phi(A, B)$.

(II) (Theorem 2.19, Hirschfeldt and Shore [9]) If $G$ is Mathias 2-generic over $\mathcal{M}$, then there is no $B \in \mathcal{M}[G]$ such that $\mathcal{M}[G] \models \Phi(A, B)$.

Theorem 2.2. Let $\mathcal{A}$ and $\mathcal{B}$ be computable directed graphs.

(I) If $U$ and $V$ are sets such that $V$ is Cohen 2-U-generic, then

$$
\mathcal{A} \cong{ }_{\operatorname{deg}(U)} \mathcal{B} \Leftrightarrow \mathcal{A} \cong{ }_{\operatorname{deg}(U \oplus V)} \mathcal{B}
$$

(II) If $U$ and $V$ are sets such that $V$ is Mathias 2-U-generic, then

$$
\mathcal{A} \cong{ }_{\operatorname{deg}(U)} \mathcal{B} \Leftrightarrow \mathcal{A} \cong{ }_{\operatorname{deg}(U \oplus V)} \mathcal{B}
$$

Proof. The left-to-right implications are trivial. To prove the right-to-left directions, assume that $\mathcal{A} \varlimsup_{\operatorname{deg}(U)} \mathcal{B}$. Consider the $\omega$-model $\mathcal{M}_{U}$ of $\mathrm{RCA}_{0}$ generated by $U$. Because $\mathcal{A} \varlimsup_{\operatorname{deg}(U)} \mathcal{B}$, there is no $f \in \mathcal{M}$ such that $\mathcal{M}_{U} \models \Phi_{\text {iso }}(\mathcal{A} \oplus \mathcal{B}, f)$. Applying Theorem 2.1 (I) or (II), depending on the type of forcing, we conclude that there is no $f \in \mathcal{M}_{U}[V]=\mathcal{M}_{U \oplus V}$ such that $\mathcal{M}_{U \oplus V}=\Phi_{\text {iso }}(\mathcal{A} \oplus \mathcal{B}, f)$. Hence $\mathcal{A} \varlimsup_{\operatorname{deg}(U \oplus V)} \mathcal{B}$ as required. 
Corollary 2.3. Every Cohen 2-generic degree and every Mathias 2-generic degree is low for isomorphism. Furthermore, if $\mathbf{d}$ is low for isomorphism, then there is a low-for-isomorphism degree $\mathbf{c}>\mathbf{d}$. Furthermore, for any $n$, we can ensure that $\mathbf{c}^{\prime} \geq \mathbf{0}^{(\mathbf{n})}$.

Proof. For the first statement, apply Theorem 2.2 with $U$ computable and $V$ any Cohen or Mathias 2-generic set. For the second statement, fix $D \in \mathbf{d}$, let $V$ be Cohen (or Mathias) 2- $D$-generic and let $\mathbf{c}=\operatorname{deg}(D \oplus V)$. Since $V$ is generic relative to $D, \mathbf{c}>\mathbf{d}$. Let $\mathcal{A}$ and $\mathcal{B}$ be computable directed graphs. Since $\mathbf{d}$ is low for isomorphism, $\mathcal{A} \cong{ }_{\Delta_{1}^{0}} \mathcal{B}$ if and only if $\mathcal{A} \cong_{\mathbf{d}} \mathcal{B}$. By Theorem 2.2 (I) (or (II)), $\mathcal{A} \cong_{\mathbf{d}} \mathcal{B}$ if and only if $\mathcal{A} \cong_{\mathbf{c}} \mathcal{B}$. Hence, $\mathcal{A} \cong_{\Delta_{1}^{0}} \mathcal{B}$ if and only if $\mathcal{A} \cong_{\mathrm{c}} \mathcal{B}$ as required.

To show the last statement, we fix an $n$ and $D \in \mathbf{d}$. We will define a sequence of sets $X_{0}<_{T} X_{1}<_{T} \ldots$ such that $\operatorname{deg}\left(X_{n}\right)$ is low for isomorphism and $X_{n}^{\prime \prime} \leq_{T} X_{n+1}^{\prime}$ for each $n$. It will follow that $0^{(n+1)} \leq_{T} X_{n}^{\prime}$ for each $n$.

We first claim that if $Y$ is 3 - $X$-generic for $X$-computable Mathias forcing, then the principal function of $Y, p_{Y}$, dominates all functions computable in $X$. To see this, we fix an index $e$ for which $\Phi_{e}^{X}$ is computable and a condition $(F, C)$. We can $X$-computably thin $C$ to a subset $C^{\prime} \subseteq C$ such that the principal function $p_{F \cup C^{\prime}}$ dominates $\Phi_{e}^{X}$. Therefore, the set of conditions $(\widehat{F}, \widehat{C})$ for which $p_{\widehat{F} \cup \widehat{C}}$ dominates $\Phi_{e}^{X}$ is dense. Since this set of conditions is also $\Sigma_{3}^{X}$, every 3 - $X$-generic set for $X$-computable Mathias forcing must meet each such set of conditions. Our claim follows because if $p_{\widehat{F} \cup \widehat{C}}$ dominates $\Phi_{e}^{X}$ and $Y$ extends $(\widehat{F}, \widehat{C})$, then $p_{Y}$ also dominates $\Phi_{e}^{X}$.

Now the statement follows very quickly. Let $X_{0}=D$ and assume that we have defined $X_{n}$ and that $\operatorname{deg}\left(X_{n}\right)$ is low for isomorphism. Let $Y_{n}$ be 3 - $X_{n}$-generic for $X_{n}$-computable Mathias forcing. By Theorem 2.2, $\operatorname{deg}\left(X_{n} \oplus Y_{n}\right)$ is low for isomorphism as well. Now Martin's Theorem and our claim above show us that $\left(X_{n} \oplus Y_{n}\right)^{\prime} \geq_{T} X_{n}^{\prime \prime}$, and we set $X_{n+1}=X_{n} \oplus Y_{n}$.

From this corollary, we can infer the existence of low-for-isomorphism degrees with certain properties by appealing to the corresponding results for Cohen and Mathias $n$-generics for $n \geq 2$. For example, there are $\Delta_{3}^{0}$ low-for-isomorphism degrees (since there are $\Delta_{3}^{0}$ Cohen 2-generics), there are low-for-isomorphism degrees which are hyperimmune (since all Cohen 2-generic degrees are hyperimmune), there are low-for-isomorphism degrees which are not minimal (since no Cohen 2-generic degree is minimal) and there are low-for-isomorphism degrees in the jump classes $\mathbf{G L}_{\mathbf{1}}$ and $\mathbf{G H}_{\mathbf{1}}$ (since Cohen 2-generic degrees are in $\mathbf{G L}_{\mathbf{1}}$ and Mathias 2-generic degrees are in $\mathbf{G H}_{\mathbf{1}}$ ). We can even infer the existence of a high low-for-isomorphism degree. Furthermore, since the low-for-isomorphism degrees are closed downwards, there are low-for-isomorphism degrees in $\mathbf{G L}_{\mathbf{2}}-\mathbf{G L}_{\mathbf{1}}$ (because every Cohen 2-generic degree bounds a degree in this class) and in $\mathbf{G L}_{\mathbf{3}}-\mathbf{G L}_{\mathbf{2}}$ (because every Cohen 3-generic degree bounds a degree in this class).

We turn to Sacks forcing with computable perfect trees to obtain low-for-isomorphism degrees which are minimal and hyperimmune free. Although various people have observed that Sacks forcing can be used in this context, there does not appear to be a proof in the literature. We review the relevant definitions and lemmas, but refer the reader to Chapter V.5 in Odifreddi [16] for the proofs of the computational lemmas. We use $\lambda$ to denote the 
empty string, $\alpha \sqsubseteq \beta$ to denote that the string $\alpha$ is an initial segment of $\beta$ and $\alpha * \beta$ (or $\alpha * n$ if $\beta=\langle n\rangle$ ) to denote the concatenation of $\alpha$ and $\beta$.

Definition 2.4. Let $\alpha, \beta, \gamma \in 2^{<\omega}$. We say $\beta$ and $\gamma$ split $\alpha$ if $\alpha \sqsubseteq \beta, \alpha \sqsubseteq \gamma$ and $\beta$ and $\gamma$ are incomparable. We say $\beta$ and $\gamma$ e-split $\alpha$ if $\beta$ and $\gamma$ split $\alpha$ and there is an $x$ such that $\Phi_{e}^{\beta}(x) \downarrow \neq \Phi_{e}^{\gamma}(x) \downarrow$.

Definition 2.5. A computable perfect tree is a computable function $T: 2^{<\omega} \rightarrow 2^{<\omega}$ such that for all $\sigma, T(\sigma * 0)$ and $T(\sigma * 1)$ split $T(\sigma)$. We say that a string $\tau$ is on $T$ if $\tau=T(\sigma)$ for some $\sigma$. We say that a set $A$ is on $T$ if for all $n$, there is an $m \geq n$ such that $A\lceil m$ is on $T$.

Definition 2.6. Let $T$ be a computable perfect tree. $S$ is a computable perfect subtree of $T$ if $S$ is a computable perfect tree and for all $\sigma, S(\sigma)=T(\tau)$ for some string $\tau$. For any string $\delta$, the full subtree of $T$ above $\delta$ is the subtree $S$ defined by $S(\sigma)=T(\delta * \sigma)$ for all $\sigma$.

To construct a noncomputable hyperimmune-free degree by forcing with computable perfect trees, we use the following two standard lemmas.

Lemma 2.7. For any computable perfect tree $T$ and any index e, there is a computable perfect subtree $S$ of $T$ such that for all $A$ on $S, A \neq \Phi_{e}$.

Lemma 2.8. For any computable perfect tree $T$ and any index e, there is a computable perfect subtree $S$ of $T$ such that either $\Phi_{e}^{A}$ is not total for all $A$ on $S$, or $\Phi_{e}^{A}$ is total for all $A$ on $S$ and $\Phi_{e}^{S(\sigma)}(n) \downarrow$ for all $n \leq|\sigma|$. In the latter case, for all $A$ on $S, \Phi_{e}^{A}$ is majorized by the computable function $f(n)=\max \left\{\Phi_{e}^{S(\sigma)}(n)|| \sigma \mid=n\right\}$.

Definition 2.9. A computable perfect tree is e-splitting if for all $\sigma, T(\sigma * 0)$ and $T(\sigma * 1)$ $e$-split $T(\sigma)$.

To construct a minimal degree by forcing with computable perfect trees, we use the following standard lemma.

Lemma 2.10. For any computable perfect tree $T$ and any index e, there is a computable perfect subtree $S$ of $T$ such that either

- for every $A$ on $S$, if $\Phi_{e}^{A}$ is total, then $\Phi_{e}^{A}$ is computable, or

- for every $A$ on $S$, if $\Phi_{e}^{A}$ is total, then $A \leq_{T} \Phi_{e}^{A}$.

Theorem 2.11. There is a degree $\mathbf{d}$ such that $\mathbf{d}$ is hyperimmune free, minimal and low for isomorphism.

Proof. For this construction, we use forcing with perfect trees. We build a (noneffective) sequence of computable perfect trees

$$
T_{0} \supseteq T_{1} \supseteq T_{2} \supseteq \cdots
$$

such that $T_{0}$ is the identity tree, $T_{i+1}$ is a computable perfect subtree of $T_{i}$, and $T_{i}(\lambda) \subsetneq T_{i+1}(\lambda)$ for all $i$. We set $D$ to be the unique set such that $T_{i}(\lambda) \sqsubseteq D$ for all $i$ and let $\mathbf{d}$ be the degree of 
$D$. We will have four types of requirements in order to make $\mathbf{d}$ noncomputable, hyperimmune free, minimal and low for isomorphism. The first three parts are standard and we mention them only briefly.

To make $\mathbf{d}$ noncomputable, hyperimmune free and minimal, we meet the requirements

$$
\operatorname{Diag}_{e}: D \neq \Phi_{e}
$$

HIFree $_{e}: \Phi_{e}^{D}$ is not total or $\Phi_{e}^{D}$ is majorized by a computable function, and

$$
\operatorname{Min}_{e}: \Phi_{e}^{D} \text { total } \rightarrow\left(\Phi_{e}^{D} \text { is computable or } D \leq_{T} \Phi_{e}^{D}\right)
$$

for each $e$. Depending on which requirement has highest priority at stage $s+1$, we apply Lemma $2.7,2.8$ or 2.10 to $T_{s}$ to obtain $T_{s+1}$.

We must now explain how to force $\mathbf{d}$ to be low for isomorphism. Fix a (noneffective) list $\left(\mathcal{A}_{i}, \mathcal{B}_{i}\right), i \in \omega$, of all pairs of infinite computable directed graphs. (We assume without loss of generality that the domains of $\mathcal{A}_{i}$ and $\mathcal{B}_{i}$ are $\omega$.) We meet the requirements

$$
\operatorname{Low}_{\langle e, i\rangle} \text { : If } \Phi_{e}^{D} \text { is an isomorphism } \mathcal{A}_{i} \rightarrow \mathcal{B}_{i} \text {, then } \mathcal{A}_{i} \cong{ }_{\Delta_{1}^{0}} \mathcal{B}_{i} \text {. }
$$

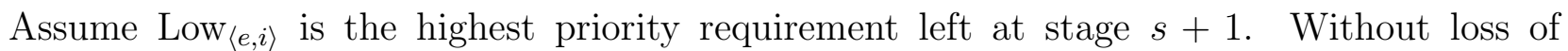
generality, we assume that we satisfy the requirement HIFree $_{e}$ before working on Low $\langle e, i\rangle$ for any $i$. If we satisfied HIFree $_{e}$ by guaranteeing that $\Phi_{e}^{D}$ is not total, then $\operatorname{Low}_{\langle e, i\rangle}$ is also satisfied. Assume we satisfied HIFree $e_{e}$ by guaranteeing that $\Phi_{e}^{D}$ is total and majorized by a computable function. In this case, for all $A$ on $T_{s}, \Phi_{e}^{A}$ is total. We proceed in two steps.

Step 1. Check (noneffectively) whether there is a string $\sigma$ and a number $n$ such that $\Phi_{e}^{T_{s}(\sigma)}\left\lceil n \downarrow\right.$ and $\Phi_{e}^{T_{s}(\sigma)} \uparrow n$ is not a partial isomorphism from $\mathcal{A}_{i}$ to $\mathcal{B}_{i}$. If there is such a string $\sigma$, then define $T_{s+1}$ to be the full subtree of $T_{s}$ above $\sigma$, skip Step 2 below and proceed to the next stage. In this case, for any $A$ on $T_{s+1}$ we have that $\Phi_{e}^{A} \uparrow n=\Phi_{e}^{T_{s}(\sigma)}\left\lceil n\right.$ and hence $\Phi_{e}^{A}$ is not an isomorphism from $\mathcal{A}_{i}$ to $\mathcal{B}_{i}$. In particular, we have satisfied Low $\langle e, i\rangle$. If there is no such string $\sigma$, proceed to Step 2 .

Step 2. Check (noneffectively) whether there is a string $\sigma$ and a number $m$ such that for all strings $\tau \sqsupseteq \sigma$ and all numbers $x, \Phi_{e}^{T_{s}(\tau)}(x) \neq m$ (either by failing to converge or converging to a number other than $m$ ). If there is such a string $\sigma$, then define $T_{s+1}$ to be the full subtree of $T_{s}$ above $\sigma$. In this case, we have guaranteed that for each $A$ on $T_{s+1}, m$ is not in the range of $\Phi_{e}^{A}$. Thus, we have satisfied $\operatorname{Low}_{\langle e, i\rangle}$ and can proceed to stage $s+1$.

If there is no such string $\sigma$ and number $m$, then we want to define $T_{s+1}$ to be a computable subtree of $T_{s}$ such that for all $A$ on $T_{s+1}, \Phi_{e}^{A}$ is onto. We define $T_{s+1}(\tau)$ by induction on the length of $\tau$. Set $T_{s+1}(\lambda)=T_{s}(\langle 0\rangle)$. For the inductive case, assume $|\tau|=m, T_{s+1}(\tau)$ is defined and $T_{s+1}(\tau)=T_{s}(\delta)$. For $i \in\{0,1\}$, we computably search for a string $\sigma_{i}$ and a number $x$ such that $\delta * i \sqsubseteq \sigma_{i}$ and $T_{e}^{T_{s}\left(\sigma_{i}\right)}(x)=m$. By our case assumptions, this search must terminate. Set $T_{s+1}(\tau * i)=T_{s}\left(\sigma_{i}\right)$.

It remains to be shown that in this final case we have satisfied $\operatorname{Low}_{\langle e, i\rangle}$. Notice that $T_{s+1}$ is a computable perfect tree with the following properties.

- For every $A$ on $T_{s+1}, \Phi_{e}^{A}$ is total (by our action for HIFree ${ }_{e}$ ).

- For every $A$ on $T_{s+1}, \Phi_{e}^{A}$ is onto (by our failure to find a string $\sigma$ in Step 2). 
- For every string $\sigma$ and number $n$, if $\Phi_{e}^{T_{s+1}(\sigma)} \uparrow(n+1) \downarrow$, then $\Phi_{e}^{T_{s+1}(\sigma)} \uparrow(n+1)$ is a partial isomorphism from $\mathcal{A}_{i}$ to $\mathcal{B}_{i}$ (by our failure to find a string $\sigma$ in Step 1).

We claim that there is a computable isomorphism between $\mathcal{A}_{i}$ to $\mathcal{B}_{i}$, and hence we have satisfied $\operatorname{Low}_{\langle e, i\rangle}$. Let $A$ be any computable path through $T_{s+1}$ (which exists because $T_{s+1}$ is a computable perfect tree). By the properties above, $\Phi_{e}^{A}$ is total, onto and for every $n$, $\Phi_{e}^{A} \uparrow(n+1)$ is a partial isomorphism. It follows that $\Phi_{e}^{A}$ is an isomorphism from $\mathcal{A}_{i}$ onto $\mathcal{B}_{i}$. Since $A$ is computable, we have $\mathcal{A}_{i} \cong{ }_{\Delta_{1}^{0}} \mathcal{B}_{i}$ as required.

\section{Degrees which are not low for isomorphism}

Having constructed examples of degrees which are low for isomorphism and have various other properties, we turn to constructing examples which are not low for isomorphism. The theme connecting these results is that if $\mathbf{d}$ bounds a degree containing a set which can be nicely approximated in some sense, then it should be possible to use this approximation to diagonalize and build a pair of computable graphs which are not computably isomorphic but are $\mathbf{d}$-computably isomorphic.

Theorem 3.1. If $\mathbf{d}$ is a noncomputable $\Delta_{2}^{0}$ degree, then $\mathbf{d}$ is not low for isomorphism. Hence, no degree which bounds a noncomputable $\Delta_{2}^{0}$ degree is low for isomorphism.

Proof. Let $D$ be a set of degree $\mathbf{d}$ and fix a $\Delta_{2}^{0}$ approximation $\left\langle D_{s}\right\rangle$ to $D$. We assume that $D_{0}=\emptyset$. We build a pair of computable directed graphs $G$ and $H$ such that there is a unique isomorphism $\alpha: G \rightarrow H$ and this isomorphism satisfies $\alpha \equiv_{T} D$.

We begin by placing a single $(n+2)$-cycle in each of $G$ and $H$ for each $n$. Let $x_{n}$ denote a fixed element of the $(n+2)$-cycle in $G$ and add an element $a_{n}$ with an edge from $x_{n}$ to $a_{n}$. Similarly, let $y_{n}$ denote a fixed element of the $(n+2)$-cycle in $H$ and add an element $b_{n}$ with an edge from $y_{n}$ to $b_{n}$. For a fixed $n$, we refer to these components as the $n$-th components of $G$ and $H$ respectively. We can visualize these $n$-th components side by side as follows:

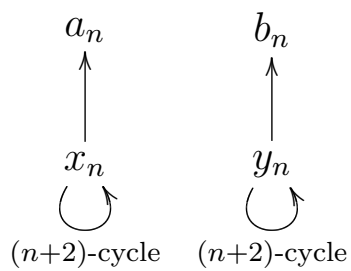

Throughout the construction, we maintain the property that there is a unique isomorphism between $G$ and $H$ and that this isomorphism matches up $n$-th components and sends $x_{n}$ to $y_{n}$. When $n \notin D_{s}$, the isomorphism will send $a_{n}$ to $b_{n}$, while if $n \in D_{s}$, the isomorphism will not map $a_{n}$ to $b_{n}$. More specifically, the $n$-th components of $G$ and $H$ remain the same until the first stage $s^{\prime}$ (if any) at which $n \in D_{s^{\prime}}$. At stage $s^{\prime}$, add new elements $a^{\prime}$ and $b^{\prime}$ 
(respectively) to the $n$-th component of $G$ and $H$ as follows.
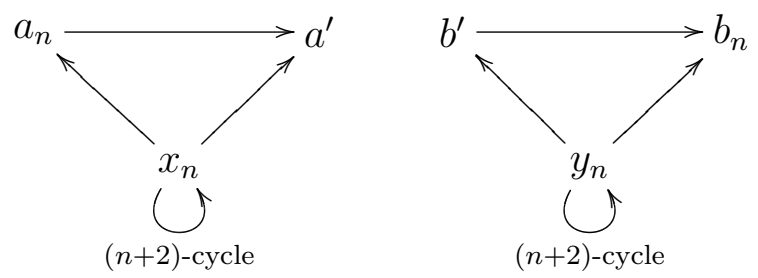

The unique isomorphism between $G$ and $H$ now sends $a_{n}$ to $b^{\prime}$ and $a^{\prime}$ to $b_{n}$. We leave the $n$-th components unchanged until the next stage $s^{\prime \prime}$ (if any) at which $n \notin D_{s^{\prime \prime}}$. At stage $s^{\prime \prime}$, add new elements $a^{\prime \prime}$ and $b^{\prime \prime}$ to the $n$-th components as follows.
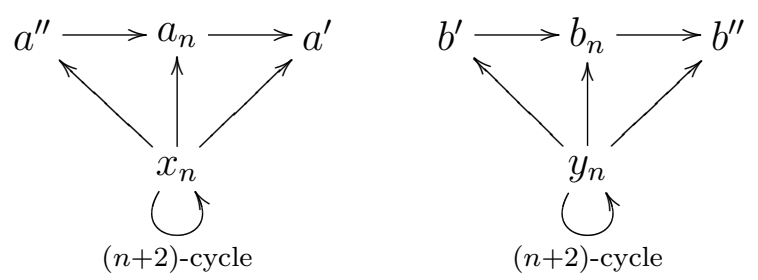

We have restored the property that the unique isomorphism sends $a_{n}$ to $b_{n}$. From here, the pattern repeats. Each time $n$ enters $D_{s}$, we add a new element to each of the linear chains above $x_{n}$ and $y_{n}$ to ensure that $a_{n}$ cannot map to $b_{n}$. When $n$ leaves $D_{s}$, we add an element to each linear chain to ensure that $a_{n}$ maps to $b_{n}$ once again. Because $D$ is $\Delta_{2}^{0}$, such changes occur only finitely often for each $n$. Once $D_{s}$ has stopped changing on $n$, the $n$-th components of $G$ and $H$ stabilize and the unique isomorphism maps $a_{n}$ to $b_{n}$ if and only if $n \notin D$. Therefore, for any degree $\mathbf{a}, G \cong{ }_{\mathbf{a}} H$ if and only if $\mathbf{d} \leq \mathbf{a}$.

Corollary 3.2. There are hyperimmune degrees, minimal degrees and $\boldsymbol{G L}_{1}$ degrees which are not low for isomorphism.

Proof. These statements follow from Theorem 3.1 because every nonzero $\Delta_{2}^{0}$ degree is hyperimmune, there are minimal $\Delta_{2}^{0}$ degrees, and there are low $\Delta_{2}^{0}$ degrees.

Corollary 3.3. The low-for-isomorphism degrees are not closed under join.

Proof. There are Cohen 2-generic degrees $\mathbf{a}$ and $\mathbf{b}$ such that $\mathbf{a} \cup \mathbf{b} \geq \mathbf{0}^{\prime}$.

We can now observe that our result concerning Cohen 2-generics cannot be strengthened. It is clear that there are Cohen 1-generics that are not low for isomorphism since there are $\Delta_{2}^{0}$ Cohen 1-generics. Furthermore, there are Cohen weak 2-generics that are not low for isomorphism because there are Cohen weak 2-generics above $\mathbf{0}^{\prime}$.

Theorem 3.4. Let $X$ and $Y$ be any pair of computably inseparable c.e. sets. No degree $\mathbf{d}$ which can compute a separating set for $X$ and $Y$ is low for isomorphism. 
Proof. The proof is similar to the proof of Theorem 3.1. Fix $X$ and $Y$ with their c.e. approximations $\left\langle X_{s}\right\rangle$ and $\left\langle Y_{s}\right\rangle$. We construct a pair of computable directed graphs $G$ and $H$ such that every isomorphism between $G$ and $H$ can compute a separating set for $X$ and $Y$ and such that every separating set for $X$ and $Y$ can compute such an isomorphism.

At the start of the construction, for each $n \in \omega, G$ and $H$ each contain an $(n+2)$-cycle (called the $n$-th components). Let $x_{n}$ (respectively $y_{n}$ ) denote a fixed element of the $(n+2)$ cycle in $G$ (in $H$ ). Add two elements $a_{n}$ and $a_{n}^{\prime}$ (respectively $b_{n}$ and $b_{n}^{\prime}$ ) to the $n$-th component of $G$ (of $H$ ) with edges from $x_{n}$ to $a_{n}$ and $a_{n}^{\prime}$ (from $y_{n}$ to $b_{n}$ and $b_{n}^{\prime}$ ). We can visualize these $n$-th components side by side as follows:
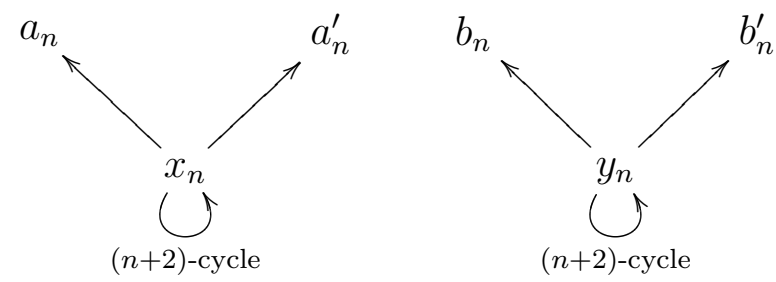

As the construction proceeds, we maintain the property that any isomorphism between $G$ and $H$ must match up $n$-th components and send $x_{n}$ to $y_{n}$. However, there may be more than one way to match up $a_{n}$ and $a_{n}^{\prime}$ with $b_{n}$ and $b_{n}^{\prime}$ and these choices are independent for each $n$. At the start, there are two options for each $n$; we can map $a_{n}$ to $b_{n}$ and $a_{n}^{\prime}$ to $b_{n}^{\prime}$ or map $a_{n}$ to $b_{n}^{\prime}$ and $a_{n}^{\prime}$ to $b_{n}$. As long as $n \notin X_{s} \cup Y_{s}$, we continue to allow both options. However, if $n$ enters $X_{s}$, we add elements $a^{\prime \prime}$ and $b^{\prime \prime}$ as follows:
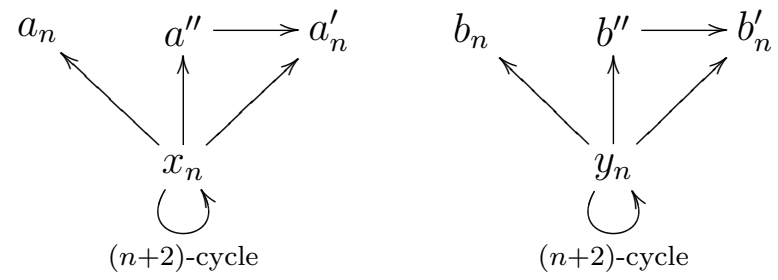

This forces the isomorphism to send $a_{n}$ to $b_{n}$. On the other hand, if $n$ enters $Y_{s}$, we add elements $a^{\prime \prime}$ and $b^{\prime \prime}$ as follows:
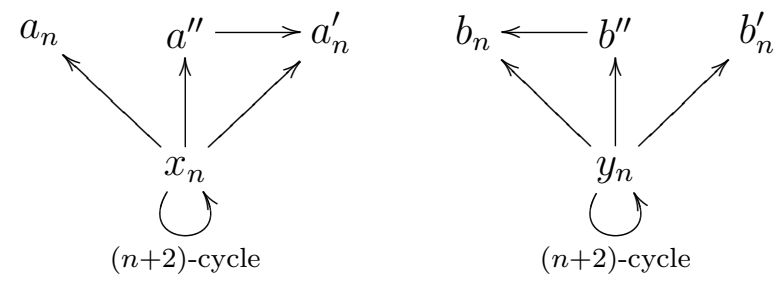

This forces the isomorphism to send $a_{n}$ to $b_{n}^{\prime}$.

If $\alpha: G \rightarrow H$ is an isomorphism (at the end of the construction), then $\left\{n \mid \alpha\left(a_{n}\right)=b_{n}\right\}$ contains $X$ and is disjoint from $Y$. Therefore, if $\mathbf{c}$ can compute an isomorphism, then $\mathbf{c}$ can compute a separating set, and hence $G \varlimsup_{\Delta_{1}^{0}} H$ since $X$ and $Y$ are computably inseparable.

On the other hand, suppose $S$ is a separating set. We use $S$ to define an isomorphism $\alpha: G \rightarrow H$. First, let $\alpha\left(x_{n}\right)=y_{n}$ and let $\alpha$ match the remainder of the $(n+2)$-cycles in $G$ 
and $H$. If $n \in S$, set $\alpha\left(a_{n}\right)=b_{n}$ and $\alpha\left(a_{n}^{\prime}\right)=b_{n}^{\prime}$. If $n \notin S$, set $\alpha\left(a_{n}\right)=b_{n}^{\prime}$ and $\alpha\left(a_{n}^{\prime}\right)=b_{n}$. Note that if $n \notin X \cup Y$, then these conditions completely define $\alpha$ as an isomorphism between the $n$-th components. If $n \in X \cup Y$, then we define $\alpha\left(a^{\prime \prime}\right)=b^{\prime \prime}$ when the elements $a^{\prime \prime}$ and $b^{\prime \prime}$ enter $G$ and $H$. Because $n \in X$ implies $n \in S$ and because $n \in Y$ implies $n \notin S$, these conditions determine an isomorphism between the $n$-th components as required.

Corollary 3.5. There are hyperimmune-free degrees which are not low for isomorphism.

Proof. This corollary follows from the Hyperimmune-Free Basis Theorem for $\Pi_{1}^{0}$ classes.

\section{Measure}

By the results of Sections 2 and 3, we know that the low-for-isomorphism degrees are large in the sense of category and that these degrees neither contain nor are disjoint from the minimal degrees, the hyperimmune-free degrees or the $\mathbf{G L}_{1}$ degrees. In this section, we show that the low-for-isomorphism degrees are small in the sense of measure and are disjoint from the Martin-Löf random degrees.

Theorem 4.1. The set of degrees which are low for isomorphism has measure 0. Furthermore, no Martin-Löf random degree is low for isomorphism.

The rest of this section is devoted to the proof of this theorem. As noted in Section 1, to show that the set of degrees which are not low for isomorphism has measure 1, it suffices to show that this set of degrees has positive measure. In the construction below, we build a $\Pi_{1}^{0}$ class $\mathcal{C}$ of positive measure such that for every $X \in \mathcal{C}$, the degree of $X$ is not low for isomorphism. To obtain the result for Martin-Löf random degrees, we apply the following theorem. ${ }^{2}$

Theorem 4.2 (Kučera [12]). Let $\mathcal{C}$ be a $\Pi_{1}^{0}$ class of positive measure. For every Martin-Löf random $A$, there is a string $\sigma \in 2^{<\omega}$ and a set $X \in \mathcal{C}$ such that $A=\sigma * X$.

By Kučera's Theorem, every Martin-Löf random computes a set in $\mathcal{C}$. Since the degrees which are not low for isomorphism are closed upwards, it follows that no Martin-Löf random degree is low for isomorphism.

For the main construction, we build (classically) isomorphic computable directed graphs $G$ and $H$ and a $\Pi_{1}^{0}$ class $\mathcal{C} \subseteq 2^{\omega}$ with the following properties.

(P1) $G \approx_{\Delta_{1}^{0}} H$

(P2) $\mu(\mathcal{C}) \geq 1 / 2$.

(P3) If $X \in \mathcal{C}$, then $X$ computes an isomorphism from $G$ to $H$.

\footnotetext{
${ }^{2}$ The authors thank Noam Greenberg for pointing out that their original nested construction of $\Pi_{1}^{0}$ classes was unnecessary to obtain the extension to Martin-Löf random degrees.
} 
Thus, $\mathcal{C}$ is a $\Pi_{1}^{0}$ class of positive measure such that the graphs $G$ and $H$ witness that every element of $\mathcal{C}$ is not low for isomorphism. To satisfy (P1), we meet the requirements

$$
R_{e}: \Phi_{e} \text { is not an isomorphism from } G \text { to } H
$$

for each $e$. To satisfy (P2), we ensure that the diagonalization strategy for $R_{e}$ does not remove too much of the tree defining the $\Pi_{1}^{0}$ class $\mathcal{C}$. To satisfy (P3), we define a Turing functional $\Gamma$ such that for all $X \in \mathcal{C}, \Gamma^{X}: G \rightarrow H$ is an isomorphism.

An e-component in $G$ or $H$ consists of an $(e+3)$-cycle with a coding node $u$ distinguished by an edge $E(u, u)$. If $e \neq e^{\prime}$, then an $e$-component is not isomorphic to an $e^{\prime}$-component. Furthermore, given two $e$-components, there is a unique isomorphism between them and this isomorphism matches the coding nodes.

A tailed e-component consists of an e-component together with two additional nodes $x_{0}$ and $x_{1}$ and edges $E\left(u, x_{0}\right), E\left(x_{0}, x_{1}\right)$ and $E\left(x_{1}, x_{0}\right)$ where $u$ is the coding node of the $e$ component. In other words, a tailed $e$-component is an $e$-component with a disjoint 2-cycle attached to the coding node. At any stage of the construction, we can convert an $e$-component into a tailed $e$-component by attaching a 2-cycle. We refer to this process as adding a tail to the respective $e$-component. At certain points, we may refer to an $e$-component as untailed to emphasize that it does not (yet) contain a tail.

The isomorphism type of $G$ and $H$ will consist of countably many untailed $e$-components for every $e$ and, for each requirement $R_{e}$ for which we actively diagonalize, countably many tailed $e$-components. For any set $X$, we will have $G \cong_{X} H$ if and only if $X$ can compute a bijection between the coding nodes in $G$ and the coding nodes in $H$ which correctly matches the coding nodes of $e$-components with and without tails. That is, given such a bijection, $X$ can effectively extend the bijection to a full isomorphism by matching up the elements in the corresponding cycles and in the corresponding tails.

We construct $G$ and $H$ in stages with $G_{s}$ and $H_{s}$ denoting these graphs at the end of stage $s$. At stage $0, G_{0}$ and $H_{0}$ contain infinitely many untailed $e$-components for each $e$. We describe the intuition behind meeting a single $R_{e}$ and defining $\mathcal{C}$ and $\Gamma$. We consider the interaction between these strategies for a single $R_{e}$ and then give the full construction.

To meet a single $R_{e}$, we fix an $e$-component in $G_{0}$ and use its coding node $a_{e}$ as a diagonalizing witness. If we never see a stage $s$ at which $\Phi_{e, s}\left(a_{e}\right)=b$ for some coding node $b$ of an $e$-component in $H_{s}$, then $R_{e}$ is trivially satisfied. If $\Phi_{e, s}\left(a_{e}\right)=b$ for such a coding node $b$, then we actively diagonalize by adding tails to an infinite coinfinite set of $e$-components in $H_{s}$ including the $e$-component coded by $b$. To maintain isomorphic structures, we also add tails to an infinite coinfinite set of $e$-components in $G_{s}$ but we do not add a tail to the $e$-component coded by $a_{e}$ in $G_{s}$. This action meets $R_{e}$, and we will not change the $e$-components in either structure after this stage. Note that all the components in $G$ and $H$ exist at stage 0 and the only change is to add tails to some of these components.

We define the $\Pi_{1}^{0}$ class $\mathcal{C}$ by building a computable sequence of trees $T_{s} \subseteq 2^{<\omega}$ such that $2^{<\omega}=T_{0} \supseteq T_{1} \supseteq \cdots$. We let $T=\cap_{s} T_{s}$ and define $\mathcal{C}=[T]$. (Recall that for any $\sigma \in 2^{<\omega}$, we define $[\sigma]$ to be the set of infinite binary strings $X$ extending $\sigma$ and for any subset $S$ of $2^{<\omega}$, we define $[S]$ to be the set of infinite binary strings $X$ extending some $\sigma \in S$.) At stage $s$, we say that we remove a string $\sigma$ from $T$ to mean that $\sigma \notin T_{s}$ and hence implicitly that $\tau \notin T_{s}$ 
for all $\tau$ extending $\sigma$. When removing $\sigma$ from $T$ at stage $s$, we do not assume that $\sigma \in T_{s-1}$. That is, we could have $\sigma \notin T_{s-1}$ because some initial segment of $\sigma$ was removed from $T$ at an earlier stage.

At stage 0 , we define the Turing functional $\Gamma$ so that for all $X \in\left[T_{0}\right], \Gamma^{X}$ is an isomorphism from $G_{0}$ to $H_{0}$. More specifically, for each $e$ and each node $\sigma \in T_{0}$ with $|\sigma|=e+2$, we define $\Gamma^{\sigma}$ so that it matches up the coding nodes for $e$-components in $G_{0}$ in bijective correspondence with the coding nodes for $e$-components in $H_{0}$. For $\delta \neq \sigma$ with $|\delta|=e+2$, the matching given by $\Gamma^{\delta}$ will not be the same as the matching given by $\Gamma^{\sigma}$.

These bijective matchings extend effectively to an isomorphism between $G_{0}$ and $H_{0}$. Because the only elements added at future stages are tails to some $e$-components in $G_{s}$ or $H_{s}$, a bijective match between coding nodes for $e$-components in $G$ and $H$ will extend effectively to an isomorphism as long as it correctly matches the coding nodes for $e$-components with and without tails.

As the construction proceeds, we will need to deal with the following conflict. Suppose we see $\Phi_{e, s}\left(a_{e}\right)=b$ and add tails to some $e$-components to meet $R_{e}$. If we defined $\Gamma^{\sigma}\left(a_{e}\right)=b$ for some $\sigma \in T_{s-1}$ then the action for $R_{e}$ will also diagonalize against $\Gamma^{X}$ being an isomorphism for any $X$ extending $\sigma$. Therefore, we need to remove $\sigma$ from $T$, which will reduce the current measure of $T$ by $2^{-|\sigma|}$. To make $\mu([T]) \geq 1 / 2$, we ensure that the strings removed from $T$ for different $R_{e}$ requirements are spread out enough to make the total measure removed sufficiently small. Specifically, each $R_{e}$ will be allowed to remove at most $2^{-(e+2)}$ much measure.

We describe the full strategy for $R_{0}$ before giving the general construction. $R_{0}$ is allowed to remove at most $1 / 4$ measure from $T$, or in other words, it is allowed to remove at most one string at level two from $T$. Let $\sigma_{i}$ for $i \leq 3$ denote the nodes at level 2 in $T_{0}=2^{<\omega}$.

At the beginning of the construction, we label the coding nodes for the 0-components in $G_{0}$ by $a_{0}$ (our distinguished diagonalizing witness) and $c_{j}^{\sigma_{i}}$ for $j \in \omega$ and $i \leq 3$. Similarly, we label the coding nodes for the 0 -components in $H_{0}$ by $b_{0, i}$ for $i \leq 3$ and $d_{j}^{\sigma_{i}}$ for $j \in \omega$ and $i \leq 3$. We view these coding nodes in columns as follows.

$G_{0}$ coding nodes:

$\begin{array}{ccccc}a_{0} & c_{0}^{\sigma_{0}} & c_{0}^{\sigma_{1}} & c_{0}^{\sigma_{2}} & c_{0}^{\sigma_{3}} \\ & c_{1}^{\sigma_{0}} & c_{1}^{\sigma_{1}} & c_{1}^{\sigma_{2}} & c_{1}^{\sigma_{3}} \\ & c_{2}^{\sigma_{0}} & c_{2}^{\sigma_{1}} & c_{2}^{\sigma_{2}} & c_{2}^{\sigma_{3}} \\ & c_{3}^{\sigma_{0}} & c_{3}^{\sigma_{1}} & c_{3}^{\sigma_{2}} & c_{3}^{\sigma_{3}} \\ & c_{4}^{\sigma_{0}} & c_{4}^{\sigma_{1}} & c_{4}^{\sigma_{2}} & c_{4}^{\sigma_{3}} \\ & \vdots & \vdots & \vdots & \vdots\end{array}$

$H_{0}$ coding nodes:

$$
\begin{array}{lllll}
b_{0,0} & d_{0}^{\sigma_{0}} & d_{0}^{\sigma_{1}} & d_{0}^{\sigma_{2}} & d_{0}^{\sigma_{3}} \\
b_{0,1} & d_{1}^{\sigma_{0}} & d_{1}^{\sigma_{1}} & d_{1}^{\sigma_{2}} & d_{1}^{\sigma_{3}} \\
b_{0,2} & d_{2}^{\sigma_{0}} & d_{2}^{\sigma_{1}} & d_{2}^{\sigma_{2}} & d_{2}^{\sigma_{3}} \\
b_{0,3} & d_{3}^{\sigma_{0}} & d_{3}^{\sigma_{1}} & d_{3}^{\sigma_{2}} & d_{3}^{\sigma_{3}} \\
& d_{4}^{\sigma_{0}} & d_{4}^{\sigma_{1}} & d_{4}^{\sigma_{2}} & d_{4}^{\sigma_{3}}
\end{array}
$$

For each $i \leq 3$, we define $\Gamma^{\sigma_{i}}$ to give a bijection between these coding nodes. First, for each infinite column other than the $i$-th column, we match the coding nodes in order.

For $\ell \neq i$, set $\Gamma^{\sigma_{i}}\left(c_{k}^{\sigma_{\ell}}\right)=d_{k}^{\sigma_{\ell}}$ for all $k \in \omega$.

Second, we match $a_{0}$ with $b_{0, i}$ by setting $\Gamma^{\sigma_{i}}\left(a_{0}\right)=b_{0, i}$. Third, we use $c_{0}^{\sigma_{i}}, c_{1}^{\sigma_{i}}$ and $c_{2}^{\sigma_{i}}$ to match with $\left\{b_{0,0}, b_{0,1}, b_{0,2}, b_{0,3}\right\} \backslash\left\{b_{0, i}\right\}$ in order of the indices and then match the remainder of the 
$i$-th infinite column in $G$ with the $i$-th infinite column in $H$ by shifting the indices.

$$
\Gamma^{\sigma_{i}}\left(c_{k}^{\sigma_{i}}\right)=\left\{\begin{array}{cc}
b_{0, k} & \text { if } k<i \\
b_{0, k+1} & \text { if } i \leq k<3 \\
d_{k-3}^{\sigma_{i}} & \text { if } 3 \leq k
\end{array}\right.
$$

To give one full picture, here is the bijection given by $\Gamma^{\sigma_{1}}$.

$$
\begin{array}{rlrl}
c_{0}^{\sigma_{0}} \mapsto d_{0}^{\sigma_{0}} & c_{0}^{\sigma_{1}} & \mapsto b_{0,0} \\
c_{1}^{\sigma_{0}} \mapsto d_{1}^{\sigma_{0}} & & a_{0} & \mapsto b_{0,1} \\
\vdots & c_{1}^{\sigma_{1}} & \mapsto b_{0,2} \\
& c_{2}^{\sigma_{1}} \mapsto b_{0,3} \\
& c_{3}^{\sigma_{1}} \mapsto d_{0}^{\sigma_{1}} \\
& c_{4}^{\sigma_{1}} & \mapsto d_{1}^{\sigma_{1}} \\
& & \vdots
\end{array}
$$

$$
\begin{aligned}
& c_{0}^{\sigma_{2}} \mapsto d_{0}^{\sigma_{2}} \\
& c_{1}^{\sigma_{2}} \mapsto d_{1}^{\sigma_{2}}
\end{aligned}
$$$$
c_{0}^{\sigma_{3}}=d_{0}^{\sigma_{3}}
$$$$
c_{1}^{\sigma_{3}}=d_{1}^{\sigma_{3}}
$$

As the construction proceeds, we wait for a stage $s$ such that $\Phi_{0, s}\left(a_{0}\right)=b$ for some coding node $b$ in a 0 -component in $H_{s}$. There are two possible cases we need to consider at such a stage, and we will act differently in each case.

For the first case, suppose that $\Phi_{e, s}\left(a_{0}\right)=d_{j}^{\sigma_{\ell}}$ for some $\ell \leq 3$ and $j \in \omega$. For each $i \neq \ell$, we have defined $\Gamma^{\sigma_{i}}\left(c_{k}^{\sigma_{\ell}}\right)=d_{k}^{\sigma_{\ell}}$ for all $k \in \omega$. Therefore, without disrupting $\Gamma^{\sigma_{i}}$ for $i \neq \ell$, we can add tails to all 0-components in $G_{s}$ with coding nodes of the form $c_{k}^{\sigma_{\ell}}$ and to all 0 -components in $H_{s}$ with coding nodes of the form $d_{k}^{\sigma_{\ell}}$. Because we add a tail to $d_{j}^{\sigma_{\ell}}$ but not to $a_{0}$, we meet $R_{e}$. However, because $\Gamma^{\sigma_{\ell}}$ matches up some coding nodes of the form $c_{k}^{\sigma_{\ell}}$ with coding nodes of the form $b_{0, k^{\prime}}$ (where $k=k^{\prime}$ or $k+1=k^{\prime}$ ), the bijection given by $\Gamma^{\sigma_{\ell}}$ no longer correctly matches coding nodes with and without tails. Therefore, we remove $\sigma_{\ell}$ from $T$ and we have permanently won $R_{e}$ at the expense of only $1 / 4$ measure.

For the second case, suppose that $\Phi_{0, s}\left(a_{0}\right)=b_{0, i}$ for some $i \leq 3$. In this case, we add a tail to $b_{0, i}$ in $H_{s}$ but do not add a tail to $a_{0}$ in $G_{s}$. This action wins $R_{e}$ but because $\Gamma^{\sigma_{i}}\left(a_{0}\right)=b_{0, i}$, the bijection given by $\Gamma^{\sigma_{i}}$ is no longer correct and we must remove $\sigma_{i}$ from $T$ losing $1 / 4$ measure. We need to add tails to other 0-components in $G_{s}$ and $H_{s}$ to ensure that each of the other bijections $\Gamma^{\sigma_{j}}$ for $j \neq i$ continues to be a bijection.

Fix such a $j \leq 3$. Consider the values of $\Gamma^{\sigma_{j}}$ on the $j$-th infinite column of coding nodes $c_{k}^{\sigma_{j}}$. $\Gamma^{\sigma_{j}}$ either maps $c_{i}^{\sigma_{j}}$ to $b_{0, i}$ (if $j>i$ ) or maps $c_{i-1}^{\sigma_{j}}$ to $b_{0, i}$ (if $j<i$ ). Fix $k_{j}$ such that $\Gamma^{\sigma_{j}}\left(c_{k_{j}}^{\sigma_{j}}\right)=b_{0, i}$. Since we add a tail to $b_{0, i}$, we must add a tail to $c_{k_{j}}^{\sigma_{j}}$ for $\Gamma^{\sigma_{j}}$ to remain correct. Now consider an arbitrary index $n \leq 3$ with $n \neq i, j$. Because $n \neq j$, we have $\Gamma^{\sigma_{n}}\left(c_{k}^{\sigma_{j}}\right)=$ $d_{k}^{\sigma_{j}}$ for all $k$. In particular, $\Gamma^{\sigma_{n}}\left(c_{k_{j}}^{\sigma_{j}}\right)=\bar{d}_{k_{j}}^{\sigma_{j}}$ so we need to add a tail to $d_{k_{j}}^{\sigma_{j}}$ for $\Gamma^{\sigma_{n}}$ to remain correct. However, we have already defined $\Gamma^{\sigma_{j}}\left(c_{k_{j}+3}^{\sigma_{j}}\right)=d_{k_{j}}^{\sigma_{j}}$ so we have to add a tail to $c_{k_{j}+3}^{\sigma_{j}}$ for $\Gamma^{\sigma_{j}}$ to remain correct. This pattern repeats. $\Gamma^{\sigma_{n}}\left(c_{k_{j}+3}^{\sigma_{j}}\right)=d_{k_{j}+3}^{\sigma_{j}}$ so we add a tail to $d_{k_{j}+3}^{\sigma_{j}}$ for the sake of $\Gamma^{\sigma_{n}}$. Then, $\Gamma^{\sigma_{j}}\left(c_{k_{j}+6}^{\sigma_{j}}\right)=d_{k_{j}+3}^{\sigma_{j}}$ forces us to add a tail to $c_{k_{j}+6}^{\sigma_{j}}$ for the sake of $\Gamma^{\sigma_{j}}$ and so on.

Therefore, for each $j \leq 3$ with $j \neq i$, we add tails to all coding nodes of the form $c_{k_{j}+3 m}^{\sigma_{j}}$ and $d_{k_{j}+3 m}^{\sigma_{j}}$ for $m \in \omega$. This action respects the definition of $\Gamma^{\sigma_{j}}$ because $\Gamma^{\sigma_{j}}\left(c_{k_{j}}^{\sigma_{j}}\right)=b_{0, i}$ and 
$\Gamma^{\sigma_{j}}\left(c_{k_{j}+3(m+1)}^{\sigma_{j}}\right)=d_{k_{j}+3 m}^{\sigma_{j}}$. It also respects $\Gamma^{\sigma_{n}}$ for $n \neq i, j$ because $\Gamma^{\sigma_{n}}\left(c_{k_{j}+3 m}^{\sigma_{j}}\right)=d_{k_{j}+3 m}^{\sigma_{j}}$. Thus, we can add tails to an infinite and coinfinite set of coding nodes in $G_{s}$ and $H_{s}$ in a manner that preserves the bijections given by $\Gamma^{\sigma_{j}}$ for $j \neq i$ and that wins $R_{e}$ at the cost of removing a single node $\sigma_{i}$ with $\left|\sigma_{i}\right|=2$ from $T$.

This completes the description of satisfying a single requirement $R_{0}$ while building $T$. The general construction proceeds by using nodes at level $e+2$ of $T$ to meet $R_{e}$. The strategy for $R_{e}$ will remove at most one node from $T$ at level $e+2$. In particular, there is no injury between $R_{e}$ strategies for different indices $e$. We now give the full construction.

At stage 0, we set up the full construction as follows. For each fixed $e \in \omega$, let $\left\langle\sigma_{e, i}\right\rangle_{i<2^{e+2}}$ be a list of the binary strings of length $e+2 . G_{0}$ will have infinitely many $e$-components with the coding nodes denoted by $a_{e}$ and $c_{j}^{\sigma_{e, i}}$ for $j \in \omega$ and $i<2^{e+2}$, and $H_{0}$ will have infinitely many $e$-components with the coding nodes denoted by $b_{e, i}$ for $i<2^{e+2}$ and $d_{j}^{\sigma_{e, i}}$ for $j \in \omega$ and $i<2^{e+2}$. For each $i<2^{e+2}$, we make the following definitions for $\Gamma^{\sigma_{e, i}}$.

$$
\begin{gathered}
\Gamma^{\sigma_{e, i}}\left(a_{e}\right)=b_{e, i} \text { and } \Gamma^{\sigma_{e, i}}\left(c_{k}^{\sigma_{e, \ell}}\right)=d_{k}^{\sigma_{e, \ell}} \text { for } \ell \neq i \text { and } k \in \omega, \text { and } \\
\Gamma^{\sigma_{e, i}}\left(c_{k}^{\sigma_{e, i}}\right)= \begin{cases}b_{e, k} & \text { if } k<i \\
b_{e, k+1} & \text { if } i \leq k<2^{e+2}-1 \\
d_{k-\left(2^{e+2}-1\right)}^{\sigma_{e+1}} & \text { if } 2^{e+2}-1 \leq k\end{cases}
\end{gathered}
$$

At stage $s>0$, we actively diagonalize for each $R_{e}$ such that $\Phi_{e, s}\left(a_{e}\right)$ converges to a coding node for an $e$-component in $H_{s-1}$ and we have not yet actively diagonalized for $R_{e}$. The action we take depends on the output of $\Phi_{e, s}\left(a_{e}\right)$.

Case 1. Suppose that $\Phi_{e}\left(a_{e}\right)=d_{j}^{\sigma_{e, i}}$ for some $j \in \omega$ and $i<2^{e+2}$. Fix the value $i$.

- Remove $\sigma_{e, i}$ from $T$.

- Add a tail to each $e$-component in $G_{s}$ with coding node $c_{\ell}^{\sigma_{e, i}}$ for $\ell \in \omega$.

- Add a tail to each $e$-component in $H_{s}$ with coding node $d_{\ell}^{\sigma_{e, i}}$ for $\ell \in \omega$.

Case 2. Suppose that $\Phi_{e}\left(a_{e}\right)=b_{e, i}$ for some $i<2^{e+2}$. Fix the value $i$.

- Remove $\sigma_{e, i}$ from $T$.

- Add a tail to the $e$-component in $H_{s}$ with coding node $b_{e, i}$.

- For each $j<2^{e+2}$ with $j \neq i$, let $k_{j}$ be such that $\Gamma^{\sigma_{e, j}}\left(c_{k_{j}}^{\sigma_{e, j}}\right)=b_{e, i}$.

- Add a tail to each e-component in $G_{s}$ with coding node $c_{k_{j}+\ell \cdot\left(2^{e+2}-1\right)}^{\sigma_{e, j}}$ for $\ell \in \omega$.

- Add a tail to each $e$-component in $H_{s}$ with coding node $d_{k_{j}+\ell \cdot\left(2^{e+2}-1\right)}^{\sigma, j}$ for $\ell \in \omega$.

Lemma 4.3. $G \varlimsup_{\Delta_{1}^{0}} H$.

Proof. We need to show that each $R_{e}$ is satisfied. If we never see a stage $s$ such that $\Phi_{e, s}\left(a_{e}\right)=$ $b$ for a coding node $b$ of an $e$-component in $H_{s-1}$, then $R_{e}$ is satisfied because $a_{e}$ is a coding node for an $e$-component and $\Phi_{e}\left(a_{e}\right)$ is not.

If we do see such a stage, fix the least $s$ at which $\Phi_{e, s}\left(a_{e}\right)=b$ for a coding node $b$ of an $e$-component in $H_{s-1}$. In both cases of the construction at stage $s$, the $e$-component of 
$H_{s}$ with coding node $b$ is given a tail but the $e$-component of $G_{s}$ with coding node $a_{e}$ is not given a tail. Since $R_{e}$ has diagonalized at stage $s$, it never acts again and hence every tailed $e$-component of $G$ receives its tail at stage $s$. Therefore, $a_{e}$ remains the coding location of an untailed $e$-component in $G$ while $\Phi_{e}\left(a_{e}\right)$ becomes the coding location of a tailed $e$-component in $H$ and hence $R_{e}$ is satisfied.

Lemma 4.4. The measure $\mu([T])$ is at least $1 / 2$.

Proof. Each requirement $R_{e}$ acts at most once and removes a single node $\sigma_{e, i}$ from $T$ when it acts. Since $\left|\sigma_{e, i}\right|=2^{e+2}$, the total measure removed from $[T]$ is bounded by $\sum_{e \in \omega} 2^{-e-2}=1 / 2$. Therefore, $\mu([T]) \geq 1-1 / 2=1 / 2$.

Lemma 4.5. For each $X \in[T], \Gamma^{X}$ is a bijection between the coding nodes in $G$ and $H$ which correctly matches e-components with and without tails in these graphs.

Proof. Fix $e$. We claim that for every $s$ and $X \in\left[T_{s}\right], \Gamma^{X}$ is a bijection between the coding nodes for $e$-components in $G_{s}$ and $H_{s}$ which correctly matches the coding nodes with and without tails. Because an $e$-component which has a tail in $G$ or $H$ receives this tail at some finite stage, this claim suffices to establish the lemma.

We prove the claim by induction on $s$. When $s=0$, fix an arbitrary set $X$ (since $\left.\left[T_{0}\right]=2^{\omega}\right)$ and index $e$. Fix the index $n$ such that $X \uparrow e+2=\sigma_{e, n}$. The definitions for $\Gamma^{\sigma_{e, n}}$ given at stage 0 bijectively match the coding nodes for $e$-components in $G_{0}$ with the coding nodes for $e$-components in $H_{0}$. Since no components are tailed at stage 0 , this bijection correctly matches those components with and without tails.

For the inductive case, assume the condition in the claim holds at stage $s-1$. Fix a set $X \in\left[T_{s}\right]$ and an index $e$. We split into two cases. For the first case, assume that $R_{e}$ does not act at stage $s$. In this case, no tails are added to $e$-components at stage $s$. Since $X \in\left[T_{s}\right] \subseteq\left[T_{s-1}\right]$, the induction hypothesis implies that $\Gamma^{X}$ correctly matches the coding nodes for $e$-components with and without tails in $G_{s-1}$ and $H_{s-1}$. Since no $e$-components receive a tail at stage $s$, this matching remains correct at stage $s$.

For the second case, assume that $R_{e}$ acts at stage $s$. Fix the index $n$ such that $X$ । $e+2=\sigma_{e, n}$. We split into subcases depending on whether $R_{e}$ acts in Case 1 or Case 2 of the construction. Suppose that $\Phi_{e}\left(a_{e}\right)=d_{j}^{\sigma_{e, i}}$ so $R_{e}$ acts in Case 1 of the construction. Because $X \in\left[T_{s}\right]$, we know that $\sigma_{e, n}$ was not removed from $T$ at stage $s$ and hence $n \neq i$. The $e$-components which receive tails in $G_{s}$ and $H_{s}$ are exactly those with coding nodes $c_{\ell}^{\sigma_{e, i}}$ and $d_{\ell}^{\sigma_{e, i}}$. However, since $n \neq i$, we have $\Gamma^{\sigma_{e, n}}\left(c_{\ell}^{\sigma_{e, i}}\right)=d_{\ell}^{\sigma_{e, i}}$ for all $\ell$. Hence $\Gamma^{X}$ correctly matches up the coding nodes which receive tails at stage $s$.

For the final subcase, assume that $\Phi_{e}\left(a_{e}\right)=b_{e, i}$ so $R_{e}$ acts in Case 2 of the construction. Since $X \in\left[T_{s}\right]$, we know $n \neq i$. For each $j<2^{e+2}$ with $j \neq i$, fix $k_{j}$ as in Case 2. An $e$-component receives a tail in $G_{s}$ if and only if its coding node is $c_{k_{j}+\ell\left(2^{e+2}-1\right)}^{\sigma_{e, j}}$ for some $j \neq i$ and $\ell \in \omega$. It receives a tail in $H_{s}$ if and only if its coding node is $b_{e, i}$ or $d_{k_{j}+\ell\left(2^{e+2}-1\right)}^{\sigma_{e j}}$ for some $j \neq i$ and $\ell \in \omega$. If $j \neq n$, then $\Gamma^{\sigma_{e, n}}\left(c_{k_{j}+\ell\left(2^{e+2}-1\right)}^{\sigma_{e, j}}\right)=d_{k_{j}+\ell\left(2^{e+2}-1\right)}^{\sigma_{e, j}}$. If $j=n$, then $\Gamma^{\sigma_{e, n}}\left(c_{k_{n}}^{\sigma_{e, n}}\right)=$ $b_{e, i}$ and $\Gamma^{\sigma_{e, n}}\left(c_{k+\left(2^{e+2}-1\right)}^{\sigma_{e, n}}\right)=d_{k}^{\sigma_{e, n}}$. Therefore, $\Gamma^{\sigma_{e, n}}\left(c_{k_{n}+(\ell+1)\left(2^{e+2}-1\right)}^{\sigma_{e, n}}\right)=d_{k_{n}+\ell\left(2^{e+2}-1\right)}^{\sigma_{e, n}}$ and the bijection of coding nodes for $e$-components given by $\Gamma^{\sigma_{e, n}}$ correctly matches up those receiving tails at stage $s$. 
This completes the verification of the main construction showing that the measure of the degrees which are not low for isomorphism is 1, and by the comments after the statement of Theorem 4.1, completes the proof of Theorem 4.1. We note that this result cannot be strengthened to a broader class of random degrees. While no Martin-Löf random is low for isomorphism, we observe that there are computable randoms that are low for isomorphism: every high degree contains a computably random real [15], and we know that there is a high degree that is low for isomorphism by Corollary 2.3. However, as Joe Miller pointed out to us, it appears that this construction can be generalized to show that no diagonally noncomputable degree is low for isomorphism.

\section{Low for linear order isomorphism}

In this section, we consider the notion of low for isomorphism restricted to a class of computable structures which are not computationally universal in the sense of Proposition 1.3. We reproduce the analog of Theorem 3.4 for computable linear orders. The same techniques suffice to prove the analog of Theorem 3.1 for linear orders, but, rather than give this proof, we describe a stronger result which will appear in Suggs [19].

Definition 5.1. A degree $\mathbf{d}$ is low for LO-isomorphism if for every pair of computable linear orders $L_{0}$ and $L_{1}, L_{0} \cong{ }_{\mathbf{d}} L_{1}$ if and only if $L_{0} \cong{ }_{\Delta_{1}^{0}} L_{1}$.

Theorem 5.2. Let $X$ and $Y$ be any pair of computably inseparable c.e. sets. No degree $\mathbf{d}$ which can compute a separating set for $X$ and $Y$ is low for LO-isomorphism.

Proof. Fix $X$ and $Y$. It suffices to build isomorphic computable linear orders $L_{0}$ and $L_{1}$ such that every separating set for $X$ and $Y$ can compute an isomorphism from these linear orders and every isomorphism between them can compute a separating set for $X$ and $Y$.

Let $\mathbb{Q}$ denote the countable dense linear order without endpoints and let $\mathbb{Z}$ denote the order type of the integers. $L_{0}$ and $L_{1}$ will be computably decomposable as

$$
\begin{array}{ll}
L_{0}: & \mathbb{Q}+A_{0}+\mathbb{Q}+A_{1}+\mathbb{Q}+\cdots+\mathbb{Q}+A_{n}+\mathbb{Q}+\cdots \\
L_{1}: & \mathbb{Q}+B_{0}+\mathbb{Q}+B_{1}+\mathbb{Q}+\cdots+\mathbb{Q}+B_{n}+\mathbb{Q}+\cdots
\end{array}
$$

where for each $n, A_{n} \cong B_{n}$ will either be isomorphic to $\mathbb{Z}$ or to a finite linear order. Thus any isomorphism between these linear orders has to match up each pair $A_{n} \cong B_{n}$ and has to match up the corresponding padding copies of $\mathbb{Q}$. Because $L_{0}$ and $L_{1}$ computably decompose into these forms, we know which points lie in each $A_{n}$ or $B_{n}$ component and which points lie in each $\mathbb{Q}$ component. Thus, to compute an isomorphism, it suffices to uniformly compute isomorphisms between $A_{n}$ and $B_{n}$ for each $n$. Conversely, every isomorphism computes a uniform sequence of isomorphisms between $A_{n}$ and $B_{n}$.

We build the sequence of orders $A_{n}$ and $B_{n}$ in stages as follows. At stage $0, A_{n}$ and $B_{n}$ are each a sequence of three points

$$
\begin{array}{ll}
A_{n}: & \alpha_{n}^{-1}<a_{n}<\alpha_{n}^{1} \\
B_{n}: & \beta_{n}^{-1}<b_{n}<\beta_{n}^{1} .
\end{array}
$$


At each stage $s>0$, we proceed in one of three cases. First, if $n \notin X_{s} \cup Y_{s}$, then we add a new pairs of points to each of $A_{n}$ and $B_{n}$ on the outside of the existing points. In this case, at the end of stage $s$, we have

$$
\begin{array}{ll}
A_{n}: & \alpha_{n}^{-(s+1)}<\alpha_{n}^{-s}<\cdots<\alpha_{n}^{-1}<a_{n}<\alpha_{n}^{1}<\cdots<\alpha_{n}^{s}<\alpha_{n}^{s+1} \\
B_{n}: & \beta_{n}^{-(s+1)}<\beta_{n}^{-s}<\cdots<\beta_{n}^{-1}<b_{n}<\beta_{n}^{1}<\cdots<\beta_{n}^{s}<\beta_{n}^{s+1} .
\end{array}
$$

Note that if $n \notin X \cup Y$, then $A_{n}$ and $B_{n}$ grow into copies of $\mathbb{Z}$ and it is possible for an isomorphism between $A_{n}$ and $B_{n}$ to map $a_{n}$ to $b_{n}$ or to map $a_{n}$ to an element other than $b_{n}$.

Second, if $s$ is the first stage at which $n \in X_{s}$, then we do not add any points to $A_{n}$ or $B_{n}$ at stage $s$ or at any future stages. Therefore, the final forms of $A_{n}$ and $B_{n}$ are

$$
\begin{array}{ll}
A_{n}: & \alpha_{n}^{-s}<\cdots<\alpha_{n}^{-1}<a_{n}<\alpha_{n}^{1}<\cdots<\alpha_{n}^{s} \\
B_{n}: & \beta_{n}^{-s}<\cdots<\beta_{n}^{-1}<b_{n}<\beta_{n}^{1}<\cdots<\beta_{n}^{s} .
\end{array}
$$

In this case, $A_{n}$ and $B_{n}$ have finished growing and the unique isomorphism between them maps $a_{n}$ to $b_{n}$.

Third, if $s$ is the first stage at which $n \in Y_{s}$, then we add one point on the right end of $A_{n}$ and one point on the left end of $B_{n}$. We do not add any further points to $A_{n}$ or $B_{n}$ at future stages. Therefore, the final forms of $A_{n}$ and $B_{n}$ are

$$
\begin{array}{cc}
A_{n}: & \alpha_{n}^{-s}<\cdots<\alpha_{n}^{-1}<a_{n}<\alpha_{n}^{1}<\cdots<\alpha_{n}^{s}<\alpha_{n}^{s+1} \\
B_{n}: & \beta_{n}^{-(s+1)}<\beta_{n}^{-s}<\cdots<\beta_{n}^{-1}<b_{n}<\beta_{n}^{1}<\cdots<\beta_{n}^{s} .
\end{array}
$$

In this case, $A_{n}$ and $B_{n}$ have finished growing and the unique isomorphism between them maps $a_{n}$ to $\beta_{n}^{-1}$, and hence not to $b_{n}$.

This completes the description of the construction of $L_{0}$ and $L_{1}$. By construction, if $f: L_{0} \rightarrow L_{1}$ is an isomorphism, then $\left\{n \mid f\left(a_{n}\right)=b_{n}\right\}$ is a separating set for $X$ and $Y$. Therefore, each isomorphism computes a separating set.

Conversely, if $U$ is a separating set, namely $X \subseteq U$ and $Y \cap U=\emptyset$, then we can build an isomorphism computably in $U$ by mapping $a_{n} \mapsto b_{n}$ for all $n \in U$ and $a_{n} \mapsto \beta_{n}^{-1}$ for all $n \notin U$. Since the successor and predecessor relations are uniformly computable in each $A_{n}$ and $B_{n}$ (by construction), we can effectively extend this map across each pair $A_{n}$ and $B_{n}$, and since $\mathbb{Q}$ is computably categorical, we can extend the map across each padding $\mathbb{Q}$ component. Therefore, each separating set computes an isomorphism.

A similar proof using $\mathbb{Q}$ padding blocks can be given for the following theorem.

Theorem 5.3. If $\mathbf{d}$ is a noncomputable $\Delta_{2}^{0}$ degree, then $\mathbf{d}$ is not low for LO-isomorphism.

Rather than give a proof of Theorem 5.3, we state a stronger forthcoming result.

Definition 5.4. A degree $\mathbf{d}$ is low for $\omega$-isomorphism if for every pair $L_{0}, L_{1}$ of computable copies of the linear order $\omega, L_{0} \cong_{\mathbf{d}} L_{1}$ if and only if $L_{0} \cong_{\Delta_{1}^{0}} L_{1}$.

Theorem 5.5 (Suggs [19]). If $\mathbf{d}$ is a noncomputable $\Delta_{2}^{0}$ degree, then $\mathbf{d}$ is not low for $\omega$ isomorphism.

Corollary 5.6 (Suggs [19]). A degree $\mathbf{d}$ is not low for $\omega$-isomorphism if and only if $\mathbf{d}$ bounds a noncomputable $\Delta_{2}^{0}$ degree. 


\section{Conclusion and questions}

We have discussed the relationship of lowness for isomorphism to other properties that demonstrate some sort of computational weakness such as minimality and hyperimmune-freeness, and we have found that there is no clear relationship between lowness for isomorphism and any of the properties mentioned: there are low-for-isomorphism degrees that possess each of these properties and low-for-isomorphism degrees that don't. In addition, we have found natural classes of degrees that are low for isomorphism (for instance, the Cohen 2-generics) and natural classes of degrees that are not (for instance, the Martin-Löf randoms). We observe that in each of these cases, our bound is tight: there is a weak 2-generic that is not low for isomorphism, while there is a computably random degree that is. However, we do not have a full classification of the degrees that are low for isomorphism. Just as all of the known examples of degrees of categoricity contain sets that are computably approximable in some way, it seems that the nontrivial degrees that are low for isomorphism resist computable approximability in a very strong way.

Question 6.1. Characterize the degrees that are low for isomorphism.

Perhaps the following approach to this question might be useful. Since the set of degrees of categoricity is countable and no Martin-Löf random degree is low for isomorphism, we can see that almost every degree (in terms of measure) is neither. It may make sense to try to identify classes of degrees that fall into neither of these categories.

Question 6.2. Is there a natural class of degrees that contains no degrees of categoricity and no low-for-isomorphism degrees?

One possibility may be the degrees that are Cohen 1-generic but not Cohen 2-generic: these are not generic enough to guarantee lowness for isomorphism, and they are not so computably approximable that they seem likely to be degrees of categoricity.

\section{References}

[1] Bernard A. Anderson and Barbara F. Csima, "Degrees that are not degrees of categoricity," to appear.

[2] George Barmpalias, Adam Day and Andrew Lewis, "Typicality in the Turing degrees," to appear.

[3] Peter A. Cholak, Damir D. Dzhafarov, Jeffry L. Hirst and Theodore A. Slaman, "Generics for computable Mathias forcing," to appear.

[4] Barbara F. Csima, Johanna N.Y. Franklin and Richard A. Shore, "Degrees of categoricity and the hyperarithmetic hierarchy," Notre Dame Journal of Formal Logic 54 (2013), 215231.

[5] Rodney G. Downey and Denis R. Hirschfeldt, Algorithmic Randomness and Complexity, Springer-Verlag, Heidelberg, 2010. 
[6] Ekaterina B. Fokina, Iskander Kalimullin and Russell Miller, "Degrees of categoricity of computable structures," Archive for Mathematical Logic 49 (2010), 51-67.

[7] Johanna N.Y. Franklin, "Lowness and highness properties for randomness notions," in Proceedings of the 10th Asian Logic Conference ed. T. Arai et al., World Scientific, 2010, 124-151.

[8] Denis R. Hirschfeldt, Bakhadyr Khoussainov, Richard A. Shore and Arkadii M. Slinko, "Degree spectra and computable dimensions in algebraic structures," Annals of Pure and Applied Logic 115 (2002), 71-113.

[9] Denis R. Hirschfeldt and Richard A. Shore, "Combinatorial principles weaker than Ramsey's theorem for pairs," Journal of Symbolic Logic 72 (2007), 171-206.

[10] Denis R. Hirschfeldt, Richard A. Shore and Theodore A. Slaman, "The Atomic Model Theorem and type omitting," Transactions of the American Mathematical Society 361 (2009), 5805-5837.

[11] Carl G. Jockusch, Jr., "Degrees of generic sets," in Recursion theory: its generalizations and applications ed. F.R. Drake and S.S. Wainer, Cambridge University Press, 1980, 110-139.

[12] A. Kučera, "Measure, $\Pi_{1}^{0}$ classes and complete extensions of PA," in Recursion Theory Week, Proceedings of the Conference Held at the Mathematisches Forschungsinstitut in Oberwolfach, April 15-21, 1984, ed. H.D. Ebbinghaus et al, volume 1141 Lecture Notes in Mathematics, Springer, Berlin, 1985, 245-259.

[13] Manuel Lerman, Degrees of unsolvability, Springer-Verlag, Heidelberg, 1983.

[14] André Nies, Computability and randomness, Oxford University Press, Oxford, 2009.

[15] André Nies, Frank Stephan and Sebastiaan A. Terwijn, "Randomness, relativization and Turing degrees," Journal of Symbolic Logic 70 (2005), 515-535.

[16] Piergiorgio Odifreddi, Classical recursion theory, North-Holland, Amsterdam, 1989.

[17] Stephen G. Simpson, Subsystems of second order arithmetic, Springer-Verlag, Heidelberg, 1999.

[18] R.I. Soare, Recursively enumerable sets and degrees, Springer-Verlag, Heidelberg, 1987.

[19] J. Suggs, PhD thesis, University of Connecticut, in preparation. 